\title{
IMPACTO DA HIPERTENSÃO ARTERIAL NA PREVALÊNCIA DO PÉ DIABÉTICO NO BRASIL: UMA ANÁLISE DE 10 ANOS
}

\author{
IMPACT OF HYPERTENSION ON THE PREVALENCE OF DIABETIC FOOT IN \\ BRAZIL: A 10-YEAR ANALYSIS
}

Fábio Henrique Reis Rodrigues', Letícia Scarlet Berto Santos', Lucélia Batista Neves Cunha Magalhães²

\begin{abstract}
RESUMO
Introdução: O diabetes mellitus é uma doença de alta prevalência, tanto em países em desenvolvimento quanto nos países desenvolvidos tendo como um dos seus principais fatores risco a hipertensão arterial e como uma de suas complicações vasculares o desenvolvimento do pé diabético. Objetivo: Realizar uma comparação entre a prevalência do pé diabético entre indivíduos diabéticos normotensos e indivíduos diabéticos hipertensos para definir se a hipertensão atua como fator agravador dessa complicação. Método: Estudo epidemiológico, descritivo e retrospectivo, com dados retirados do departamento de informática do sistema único de saúde (DATASUS), através do TABNET pelo programa HIPERDIA, que acompanha indivíduos hipertensos e/ou diabéticos. O período analisado foi janeiro de 2002 até janeiro de 2012, totalizando 10 anos de análise. Os dados referentes às variáveis "diabetes tipo 01" e "diabetes tipo 02" foram somadas, formando o grupo de indivíduos diabéticos normotensos. E os dados referentes à variável "hipertensão com diabetes" formou o grupo de indivíduos diabéticos hipertensos. Desses grupos, foi selecionado os indivíduos com pé diabéticos e esses dados foram sumarizados, a partir de cálculos de proporção, agrupados no Excel ${ }^{\circledast}$ e foi aplicado o teste exato de Fisher. Resultados: A proporção do pé diabético em indivíduos diabéticos normotensos foi de 3,14\%, já em indivíduos diabéticos hipertensos foi de 4,38\%, sendo seus dados estatisticamente significante (OR: 1,43; IC 95\%, $1,34-1,51 ; p<0,001)$. O perfil epidemiológico de diabéticos normotensos tem maior prevalência no sexo masculino, em 50-54 anos, $24,05 \%$ tabagistas e $30,41 \%$ com sobrepeso. Os diabéticos hipertensos apresentam maior prevalência no sexo feminino, em 60-64 anos, 30,48\% tabagistas e 46,51\% com sobrepeso. Conclusão: Conclui-se que a hipertensão arterial atua como um significante fator agravador para o desenvolvimento do pé diabético. Sendo assim, diante da escassez de estudos, o presente trabalho mostra-se relevante, servindo como base na compreensão dos aspectos epidemiológicos acerca da relação entre diabetes e hipertensão.
\end{abstract}

Descritores: Diabetes mellitus; Hipertensão arterial; Pé diabético; Complicações do diabetes; Perfil epidemiológico.

\section{ABSTRACT}

Introduction: Diabetes mellitus is a disease of high prevalence, both in developing and developed countries, having as one of its main risk factors hypertension and as one of its vascular complications the development of diabetic foot. Objective: To compare the prevalence of diabetic foot between normotensive diabetic and hypertensive diabetic individuals to define if hypertension acts as an aggravating factor of this complication. Method: Epidemiological, descriptive and retrospective study, with data taken from the informatics department of the single health system (DATASUS), through TABNET, where was accessed the HIPERDIA program, which follows hypertensive and / or diabetic individuals. The period analyzed was from January 2002 to January 2012, totaling 10 years of analysis. Data referring to the variables "type 01 diabetes" and "type 02 diabetes" were summed, forming the group of normotensive diabetic individuals. And data regarding the variable "hypertension with diabetes" formed the group of hypertensive diabetic individuals. From these groups, we selected individuals with diabetic foot and these data were summarized from proportion calculations, grouped in Excel ${ }^{\circledR}$ and Fisher's exact test was applied. Results: The proportion of diabetic foot in normotensive diabetic individuals was $3.14 \%$, while in hypertensive diabetic individuals it was $4.38 \%$, with statistically significant data (OR: 1.43; $95 \%$ Cl 1.34- 1.51; $p<0.001$ ). The epidemiological profile of normotensive diabetics is more prevalent among males, aged $50-54$ years, $24.05 \%$ smokers and $30.41 \%$ overweight. Hypertensive diabetics are more prevalent in females, aged 60-64 years, $30.48 \%$ smokers and $46.51 \%$ overweight. Conclusion: It is concluded that hypertension acts as a significant aggravating factor for the development of diabetic foot. Thus, given the scarcity of studies, the present study is relevant, serving as a basis for understanding the epidemiological aspects of the relationship between diabetes and hypertension.

Keywords: Diabetes Mellitus; Arterial Hypertension; Diabetic Foot; Diabetes Complications; Epidemiological Profile.

1. Centro Universitário FTC, Salvador, BA, Brasil.

2. Curso de medicina da Faculdade de Tecnologia e Ciências, FTC, Salvador, BA, Brasil,

Correspondência: Fabioreisrodrigues@gmail.com; leticiabertoO3@gmail.com; luceliamagalhaes@terra.com.br

http://dx.doi.org/10.47870/1519-7522/202128017-13 


\section{INTRODUÇÃO}

Diabetes mellitus (DM) é um conjunto de distúrbios de ordem metabólica que possuem como característica comum a hiperglicemia mediada pela diminuição na sensibilidade das células em relação à insulina ou por conta da diminuição ou ausência desse hormônio. A hiperglicemia associada ao DM tem como origem em sua fisiopatologia, uma associação entre fatores ambientais e genéticos e resulta na alteração do metabolismo de carboidratos, proteínas e lipídios.

A DM é uma doença de alta prevalência, tanto em países em desenvolvimento quanto nos países desenvolvidos, e em 2015, a federação internacional de diabetes ${ }^{1}$ estimou que $8,8 \%$ da população mundial com idade entre 20 e 79 anos vivia com o diabetes, sendo que em 2014, computou-se que a taxa de casos de diabetes não diagnosticados em adultos seja de $46 \%$, sendo a maior parte localizados em países em desenvolvimento $(83,8 \%)^{2}$

Em 2013, a Pesquisa Nacional de Saúde (PNS), realizada pelo Instituto Brasileiro de Geografia e Estatística (IBGE) e pelo Ministério da Saúde (MS), apresentou que cerca de 6,2\% da população brasileira com 18 anos de idade ou mais referiu diagnóstico médico de diabetes, sendo de 7,0\% nas mulheres e de $5,4 \%$ nos homens, com maior taxa de diabetes $(9,6 \%)$ nos indivíduos sem instrução ou com ensino fundamental incompleto. ${ }^{3}$

O aumento da prevalência do diabetes está associado a diversos fatores, como: rápida urbanização, transição epidemiológica, transição nutricional, maior frequência de estilo de vida sedentário, maior frequência de excesso de peso, crescimento e envelhecimento populacional e, também, à maior sobrevida dos indivíduos com diabetes. ${ }^{4}$

Como de acordo com a Organização Mundial de Saúde (OMS), a glicemia elevada é o terceiro principal fator de causa de mortalidade prematura, atrás apenas do aumento da pressão arterial e uso do tabaco. Indo um pouco além nesse dado, entende-se que para o combate da mortalidade associado ao DM são necessários que haja altos custos na saúde pública com hospitalizações e tratamento tanto ambulatorial quanto o tratamento de suas complicações sistêmicas.

Na história natural do DM, alterações fisiopatológicas estão presentes antes que os valores glicêmicos atinjam níveis supranormais. A condição na qual os valores glicêmicos estão acima dos valores de referência, mas ainda abaixo dos valores diagnósticos de DM, denomina-se pré-diabetes. A resistência à insulina já está presente e, na ausência de medidas de combate aos fatores de risco modificáveis, ela evolui frequentemente para a doença clinicamente manifesta. ${ }^{5}$

A confirmação do diagnóstico de DM requer repetição dos exames alterados, idealmente o mesmo exame alterado em segunda amostra de sangue, na ausência de sintomas inequívocos de hiperglicemia. ${ }^{5}$ Pacientes com sintomas clássicos de hiperglicemia, tais como poliúria, polidipsia, polifagia e emagrecimento, devem ser submetidos à dosagem de glicemia ao acaso e independente do jejum, não havendo necessidade de confirmação por meio de segunda dosagem caso se verifique glicemia aleatória $\geq 200 \mathrm{mg} / \mathrm{dL}$. Os critérios diagnósticos para DM1 são semelhantes aos utilizados no DM2. No primeiro caso, porém, comumente a sintomatologia já chama muito mais a atenção do clínico do que no segundo caso. ${ }^{4}$
A hiperglicemia persistente está associada a complicações crônicas micro e macrovasculares, aumento de morbidade, redução da qualidade de vida e elevação da taxa de mortalidade. ${ }^{4}$ Tradicionalmente, as complicações do diabetes são categorizadas como distúrbios microvasculares e macrovasculares, que resultam em retinopatia, nefropatia, neuropatia, doença coronariana, doença cerebrovascular e doença arterial periférica, sendo essa última associada ao desenvolvimento do pé diabético e podendo estar presente em até 32,6\% dos pacientes. ${ }^{1}$ As lesões do pé diabético resultam da combinação de dois ou mais fatores de risco que atuam concomitantemente e podem ser desencadeadas tanto por traumas intrínsecos como extrínsecos, associados à neuropatia periférica (NP), à doença vascular periférica (DVP) e à alteração biomecânica. A NP crônica associada ao DM caracteriza-se pelo comprometimento das fibras sensitivas, motoras e/ou autonômicas. O componente sensitivo acarreta perda gradual da sensibilidade protetora, percepção da pressão plantar, temperatura e propriocepção. Quanto ao componente motor, este se caracteriza por hipotrofia da musculatura intrínseca do pé, que pode desencadear deformidades osteoarticulares e alterações na deambulação. Já o comprometimento autonômico reduz ou suprime a sudorese dos pés, deixando-os secos e predispostos a fissuras, além de desencadear alterações arteriovenosas. ${ }^{6}$

O diabetes tem sido responsabilizado, entretanto, por contribuir para agravos, direta ou indiretamente, no sistema musculoesquelético, no sistema digestório, na função cognitiva e na saúde mental, além de ser associado a diversos tipos de câncer. Pouca atenção tem sido dispensada às tendências globais das complicações do diabetes e ao modo como as características da morbidade associada ao diabetes têm mudado. ${ }^{7}$

Associado a esse pensamento sobre complicações do diabetes e fatores de risco, é conhecido que uma das Comorbidades mais associadas é a hipertensão arterial (HA) que consiste em uma condição clínica multifatorial caracterizada por elevação sustentada dos níveis pressóricos $\geq 140$ e/ou 90 $\mathrm{mmHg}$. Frequentemente se associa os distúrbios metabólicos, alterações funcionais e/ou estruturais de órgãos-alvo, sendo agravada pela presença de outros fatores de risco (FR), como dislipidemia, obesidade abdominal, intolerância à glicose e diabetes melito. A incidência de HA em pacientes diabéticos tipo 1 aumenta de 5\% aos 10 anos de idade, para 33\% aos 20 anos, e para $70 \%$, aos 40 anos. Cerca de $40 \%$ dos pacientes com diagnóstico recente de DM tipo 2 tem $\mathrm{HA},{ }^{8}$ além de consideração de que a prevalência de hipertensão em diabéticos é pelo menos duas vezes maior do que na população em geral. ${ }^{9}$

Nesse sentido, o trabalho tem como objetivo comparar a prevalência de pé diabético em pacientes diabéticos hipertensos com pacientes diabéticos normotensos, traçar o perfil epidemiológico dessas duas populações, através das variáveis sexo, idade, tabagismo e sobrepeso e definir os estados com maior e menor prevalência.

\section{MATERIAL E MÉTODOS}

O trabalho em questão é um estudo do tipo epidemiológico, descritivo e retrospectivo. O mesmo teve seus dados retirados do departamento de informática do sistema único 
de saúde, o DATASUS, através do TABNET, onde é acessado o programa HIPERDIA, que é um banco de cadastros e acompanhamentos de portadores de hipertensão arterial sistêmica e/ou diabetes mellitus.

Dentro dessa plataforma foram analisadas, a partir de cada estado do Brasil, as seguintes variáveis: diabetes tipo 1, diabetes tipo 2, hipertensão com diabetes e pé diabético, sendo essa última cruzada com as variáveis anteriores. Para fazer o perfil epidemiológico da amostra, foi analisada as variáveis sexo, idade, tabagismo e sobrepeso.

Dentro do programa Excel ${ }^{\circledR}$, os dados referentes às variáveis "diabetes tipo 1" e "diabetes tipo 2" foram somadas, formando o grupo de indivíduos diabéticos normotensos. E os dados referentes à variável "hipertensão com diabetes" formou o grupo de indivíduos diabéticos hipertensos. Desses dois grupos, selecionamos os indivíduos com pé diabéticos e esses dados foram sumarizados, a partir de cálculos de proporção e foi aplicado o teste exato de Fisher. Além disso, tabelas e gráficos foram gerados com esses dados com o intuito de dinamizar a exposição dos mesmos.

O período analisado foi de janeiro de 2002 até janeiro de 2012, totalizando dez anos de análise. Foram analisados dados referentes a todos os estados do Brasil. Foi utilizando como critério de inclusão apenas indivíduos cadastrados no programa HIPERDIA. E como critérios de exclusão, os indivíduos hipertensos não diabéticos e os indivíduos referentes ao Distrito federal por não ter dados suficientes ao período de análise.

O DATASUS é uma base de dados de acesso público e gratuito, sem identificação dos participantes, dispensando apreciação por Comitê de Ética em Pesquisa (CEP), conforme dispõe a Resolução do Conselho Nacional de Saúde (CNS) no 466, de 12 de dezembro de 2012.

\section{RESULTADOS}

Uma análise da população de estudo foi feita através da ótica dos estados do Brasil, em que no grupo de indivíduos diabéticos normotensos foi observado o maior valor de prevalência no estado do Rio Grande do Norte, apresentando um valor proporcional bruto, no período estudado, de 4,89\%, enquanto que foi demonstrado o menor número de pé diabético no estado do Amazonas, apresentando um valor proporcional bruto de 1,40\%. (Figura 1)

Já na análise do grupo de indivíduos diabéticos hipertensos foi observado o maior valor de prevalência novamente no Rio Grande do Norte apresentando um valor proporcional bruto, no período estudado, de $6,04 \%$, enquanto que foi demonstrado o menor número de pé diabético no estado do Amazonas, assim como no grupo de indivíduos diabéticos normotensos, apresentando um valor proporcional bruto de 1,64\%. (Figura 2)

Em seguida foi traçado o perfil epidemiológico dos dois grupos, o de indivíduos com pé diabético que são diabéticos normotensos e o de indivíduos com pé diabético que são diabéticos hipertensos. A partir dessa análise, foi visto a prevalência do sexo masculino no grupo de diabéticos normotensos, em 50,04\%, prevalência da faixa etária de 50-54 anos, 24,05\% tabagistas e 30,41\% apresentando sobrepeso como comorbidade. Já no grupo de diabéticos hipertensos, foi visto a prevalência do sexo feminino, em $64,29 \%$, prevalência na faixa etária de 60-64 anos, 30,48\% tabagistas e 46,51\% com sobrepeso. (Tabela 1)

O número total de indivíduos diabéticos normotensos em todo o Brasil totalizou 456.154 mil, sendo que desses, 14.332 mil apresentaram pé diabético como complicação (Figura 3), revelando uma proporção de 3,14\%. (Figura 5) Já o número total de casos no Brasil de indivíduos diabéticos hipertensos totalizou 2.257.966 milhões, sendo que desses, 98.902 mil apresentaram pé diabético como complicação (Figura 4), revelando uma proporção de 4,38\%. (Figura 5)

Nesse sentido, pode-se inferir um total de indivíduos diabéticos no estudo, independendo de comorbidade associada, como a soma dos indivíduos dos dois grupos, totalizando, então, 2.714.120 milhões de indivíduos. É possível estimar, também, a prevalência de hipertensão arterial na população de indivíduos com pé diabético, resultando em um número de $87,3 \%$.

Foi aplicado o teste exato de Fisher revelando um $\mathrm{p}$

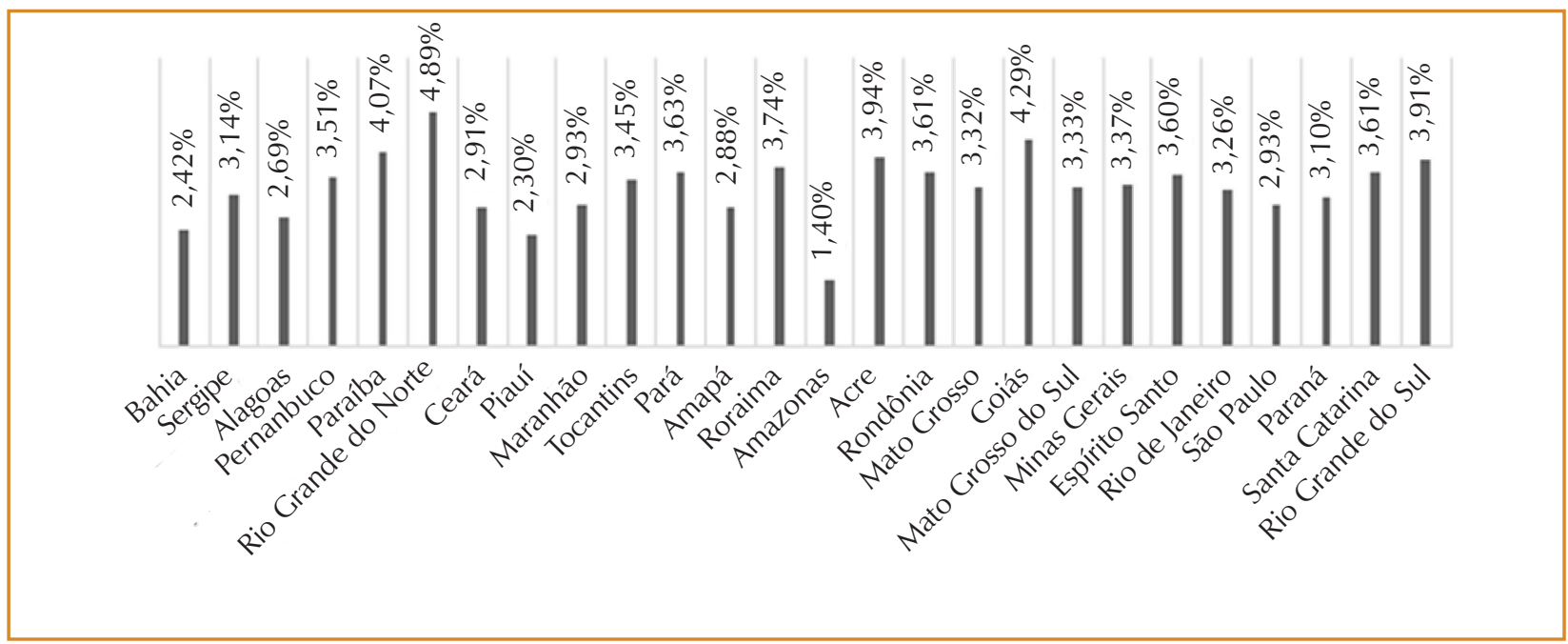

Figura 1. Proporção de indivíduos com pé diabéticos no grupo de indivíduos diabéticos normotensos nos estados do Brasil. 


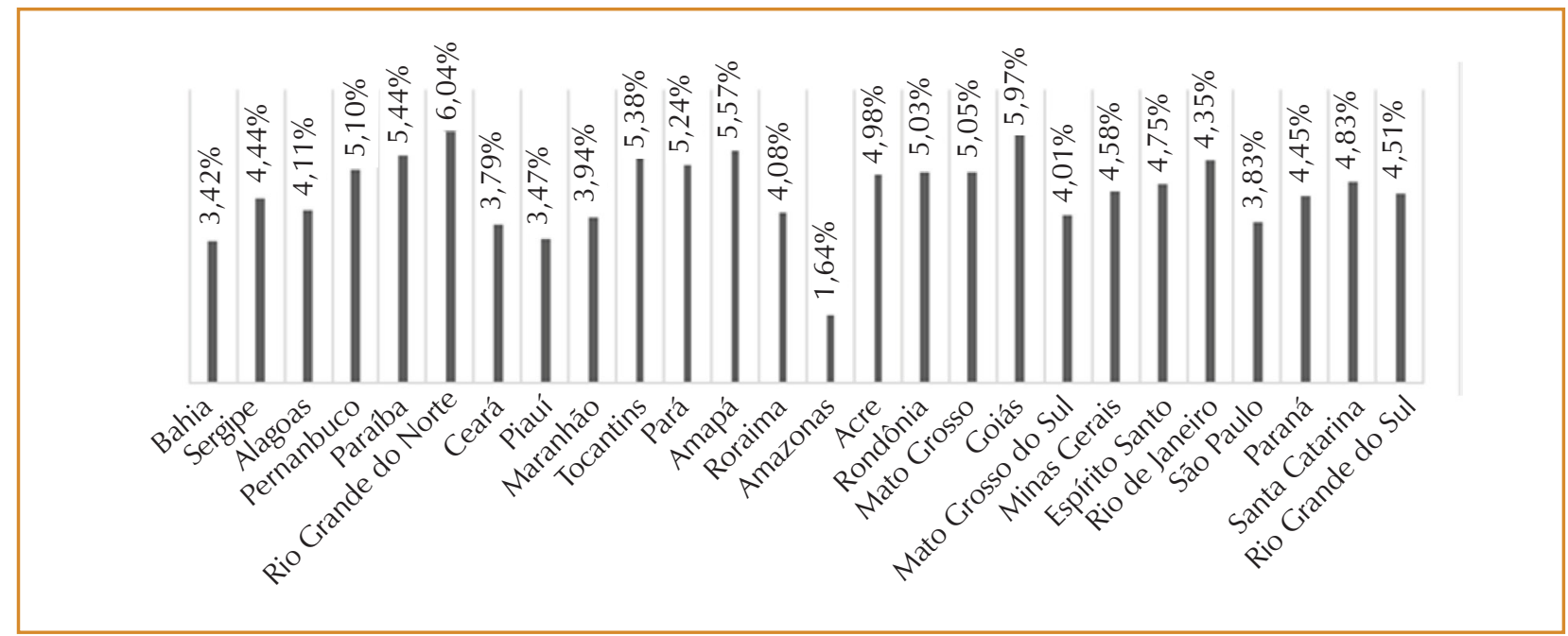

Figura 2. Proporção de indivíduos com pé diabéticos no grupo de indivíduos diabéticos hipertensos nos estados do Brasil.

Tabela 1. Perfil epidemiológico dos grupos estudados em relação ao pé diabético nos estados do Brasil.

\begin{tabular}{c|c|c} 
Variáveis & $\begin{array}{c}\text { Diabéticos } \\
\text { normotensos n (\%) }\end{array}$ & $\begin{array}{c}\text { Diabéticos } \\
\text { hipertensos n (\%) }\end{array}$ \\
\hline Sexo & $7.172(50,04 \%)$ & $35.298(35,70 \%)$ \\
\hline Masculino & $7.150(49,95 \%)$ & $63.568(64,29 \%)$ \\
\hline Feminino & $50-54$ anos (14,1\%) & $60-64$ anos $(14,7 \%)$ \\
\hline Faixa etária & $3.376(24,05 \%)$ & $27.132(30,48 \%)$ \\
\hline Tabagismo & $4.635(30,41 \%)$ & $49.233(46,51 \%)$ \\
\hline Sobrepeso & &
\end{tabular}

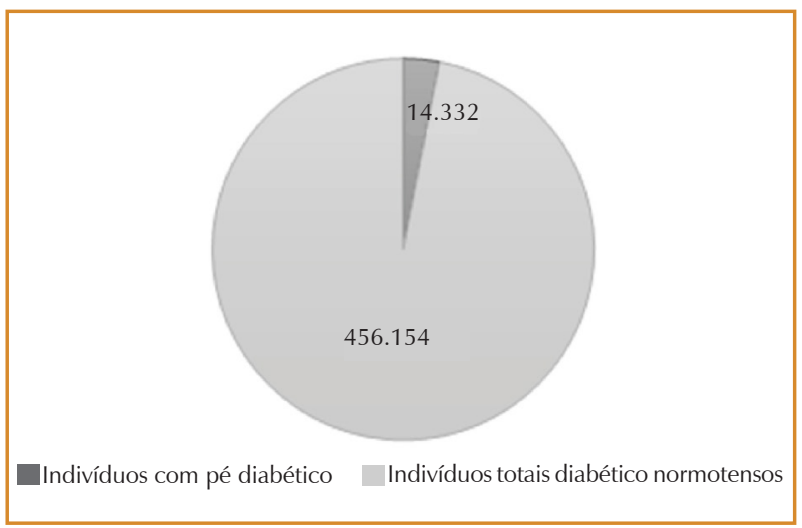

Figura 3. Número total de casos de indivíduos do grupo de diabéticos normotensos e número total de indivíduos com pé diabéticos deste grupo.

menor do que 0,001. Sendo assim, esse valor apresenta, portanto, significância estatística ao presente estudo. Foi revelado, também, uma Odds Ratio de 1,43, o que mostrou que as chances de um indivíduo do grupo de diabéticos hipertensos apresentarem pé diabético como complicação crônica do diabetes é 1,43 vezes maior do que um indivíduo diabético que não apresenta hipertensão associada. O intervalo de confiança tendo se mantido sempre acima do valor $1(1,41-1,46)$ reafirma as maiores chances dessa ocorrência. (Figura 05)

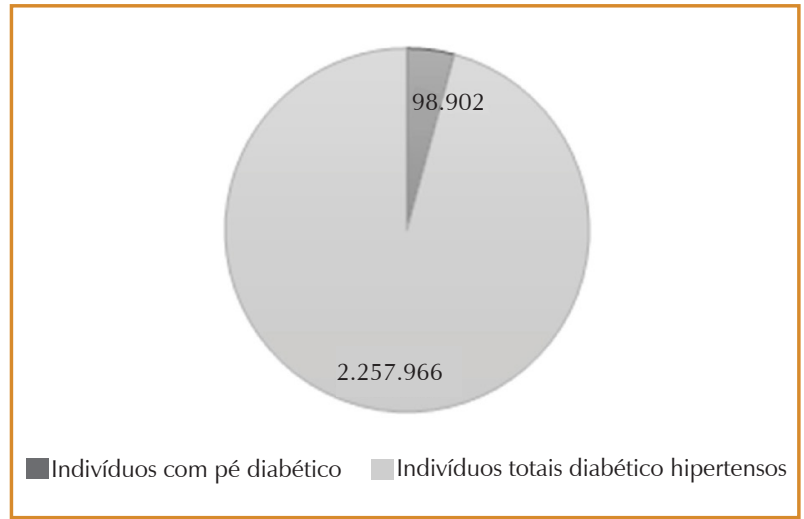

Figura 4. Número total de casos de indivíduos do grupo de diabéticos hipertensos e número total de indivíduos com pé diabéticos deste grupo.

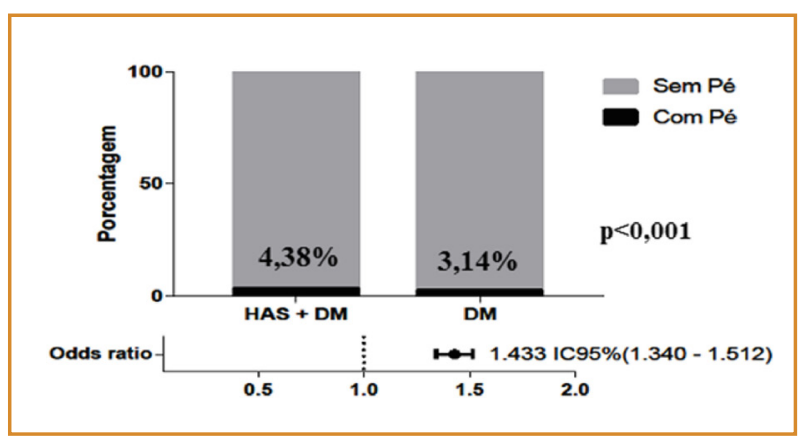

Figura 5. Proporções dos dois grupos, odds ratio intervalo de confiança da estatística aplicada.

\section{DISCUSSÃO}

Nos 10 anos de análise foi observada uma prevalência de pé diabético no sexo masculino no grupo de indivíduos diabéticos normotensos, enquanto que no grupo de indivíduos diabéticos hipertensos houve prevalência no sexo feminino, em valores de proporção de 50,04\% e 64,29\%, respectivamente. O resultado no sexo masculino está em consonância 
com Li e cols. que demonstra uma prevalência de $62,5 \%$ no sexo masculino, porém não apresentando valor de $p$ com significância, ${ }^{10}$ assim como Benjamim e cols., estudo realizado em Maceió, no estado de Alagoas (AL), em que a prevalência pelo sexo masculino foi de $57,1 \% .{ }^{11}$ Já o resultado visualizado nos diabéticos hipertensos do sexo feminino corrobora com Mané e cols. , que demonstra uma taxa (masculino/feminino) de 0,88, ${ }^{12}$ além de outros dois realizados em Sergipe (SE). ${ }^{13,14}$

As diferenças de prevalência observadas entre os sexos masculino e feminino são retratadas em estudos como um produto da presença de fatores de risco, muito mais do que como diferenças genéticas ou hormonais. É evidenciado que a prevalência em relação ao sexo é resultado da interação entre diversos fatores de risco, como obesidade, disponibilidade e acesso aos serviços de saúde, atividade física, mudança de estilo de vida, entre outros, que se configuram como condições que variam no tempo e espaço, sem que exista uma tendência clara estabelecida. ${ }^{15,16}$

Em relação à variável faixa etária, foi demonstrado maiores prevalências nas faixas etárias mais elevadas, 50-54 anos e 60-64 anos, sendo o primeiro intervalo no grupo dos pacientes diabéticos normotensos. Esse dado é corroborado pelos estudos citados anteriormente na introdução do trabalho, ${ }^{1-5}$ assim como o estudo realizado por Van e cols., em Texas, nos Estados Unidos da América (EUA) que observou a incidência aumentando com a idade tanto na Califórnia como na Holanda, com pico na faixa etária de 75 anos, apresentando significância estatística. ${ }^{17}$

Esses aumentos nos valores de prevalência e incidência em relação à variável faixa-etária são esperados uma vez que Mendonça e cols. elucidam que quanto mais avançada for a idade, maiores serão as alterações fisiológicas e, consequentemente, o aparecimento das complicações advindas do DM. ${ }^{6}$ Somado a isso, Schmidt e cols. fala sobre o aumento de comorbidades que acompanham a idade, ainda mais em países de baixa e média renda, como o Brasil, em que o enfoque preventivo continua amplamente voltado para doenças infecciosas e problemas de saúde materno-infantil, tendo poucas ações preventivas de saúde pública realizadas no intuito de desenvolver e implementar uma estratégia para controlar o aumento do diabetes mellitus, ${ }^{18}$ assim como o diagnóstico que acontece de forma muito tardio.

O tabagismo é uma condição que também se faz presente na amostra do estudo de forma significativa, variando entre 24,05 a 30,48\% entre os dois grupos analisados, tendo seu maior valor no grupo dos pacientes diabéticos hipertensos. Esses valores foram condizentes com um estudo realizado por Pace e cols. , no Hospital das Clínicas da Universidade de São Paulo, que demonstrou a presença do tabagismo em 25\% dos indivíduos. ${ }^{9}$ Essa condição ainda possui valores maiores no estudo Paquistanês realizado por Moradi e cols. em que indivíduos que evoluíram a condição de pé diabético para a amputação, onde a prevalência de tabagismo foi de $60,8 \%,{ }^{19}$ sendo possível inferir que o tabagismo pode piorar o prognóstico dos pacientes com pé diabético, levando a evolução dessa condição para a amputação de membros, sendo um fator de risco sugerido através de uma meta-análise chinesa de Liu e cols. ${ }^{20}$ Essa associação entre a significativa prevalência do tabagismo entre indivíduos com pé diabético é esperada uma vez que a doença aterosclerótica vascular periférica se manifesta precocemente entre pessoas diabéticas e tabagistas, e que o tabagismo constitui um fator agravante para a obliteração das artérias e arteríolas, uma vez que a nicotina e o alcatrão são lesivos ao endotélio vascular, provocando doenças cardiovasculares e aterosclerose, especialmente em membros inferiores. ${ }^{9,21,22}$

Uma outra variável clínica observada no estudo foi o sobrepeso que demonstrou prevalências de 30,41 a 46,51\%, e assim como foi visto no tabagismo, a maior prevalência foi no grupo dos indivíduos diabético hipertensos. Dados que corroboram com esse resultado foram vistos no estudo de Pace e cols. de Ribeirão Preto ${ }^{9}$ que demonstra que a média do índice de massa corpórea (IMC) da população do estudo é de 28, que se configura sobrepeso, assim como Gimenes e cols. de São Paulo que já apresenta uma prevalência dessa condição no valor de $24,8 \%$ para indivíduos diabéticos hipertensos e $8 \%$ em indivíduos diabéticos normotensos. ${ }^{23}$

É possível constatar carências de estudos ao se tentar observar a distribuição da prevalência do desenvolvimento do pé diabético pelos estados do país. Porém, mesmo diante de poucos estudos nacionais que possam dar suporte para essa temática, alguns estados como Pernambuco, São Paulo, Alagoas, Minas Gerais, Rio Grande do Sul e Goiás possuem estudos na tentativa de realizar um perfil epidemiológico acerca do pé diabético. ${ }^{6,9,21-26}$

Segundo dados do IBGE as regiões Norte e Nordeste são as que apresentam o maior percentual de indivíduos que dependem da saúde pública, diferente do Sudeste que se apresenta como a região de maior cobertura em relação aos planos de saúde privados. Apesar disso, os municípios das regiões Norte e especialmente Nordeste são os que apresentam menos investimento em saúde pública com recursos próprios, o que evidencia a existência de grande desigualdade de acesso e disponibilidade aos serviços de saúde, impactando diretamente nas variações de prevalência observadas entre os estados brasileiros. ${ }^{27,28}$ Além disso, é observado que nos municípios localizados em regiões, com maior escassez de recurso, há grande dificuldade de manter os profissionais de saúde na atenção básica, ${ }^{29}$ o que se configura como um problema que produz impactos nos cuidados de saúde primários, diminuindo a eficiência na intervenção precoce da história natural das doenças, como DM e HAS.

Foi observado que a média das prevalências de indivíduos com pé diabético entre o grupo de indivíduos diabéticos normotensos e o grupo de diabéticos hipertensos foi de $3,76 \%$, valor que corresponde ao achado proposto por Houtum e Lavery em que a prevalência global pode variar entre $4-10 \%$, dependendo do seu desenvolvimento socioeconômico, sendo mais prevalente em países menos desenvolvidos. Outro estudo que apontava valores próximos ao do presente estudo foi feito por Moradi e cols. , através de uma meta-análise iraniana, com prevalência de 3\%. ${ }^{19}$ Porém, essa faixa de valores encontrada no estudo corresponde à apenas, aproximadamente, metade da prevalência apontada por Vieira e cols. , um estudo na cidade do Recife, no Pernambuco, ${ }^{24}$, assim como corresponde à, aproximadamente, um terço da prevalência apontada por Afroz e cols. ${ }^{30}$

O pé diabético está presente em 3,14\% dos indivíduos do grupo de diabéticos normotensos e em 4,38\% dos indivíduos do 
grupo de diabéticos hipertensos, observando então que a prevalência foi maior no grupo que apresentou hipertensão arterial como comorbidade associada, e, em seguida foi demonstrado, através de cálculos estatísticos, a significância desses dados. Em consonância a essa observação, Gimenes e cols. evidenciou $0,9 \%$ de prevalência de indivíduos com pé diabético no grupo de diabético normotensos e 7,1\% no grupo de diabéticos hipertensos. ${ }^{23} \mathrm{E}$ como dito anteriormente, as lesões do pé diabético possuem associação em sua gênese com processos como a doença vascular periférica ${ }^{6}$ que na literatura é sabido a sua relação com a hipertensão arterial uma vez que esta é o fator mais potente no desenvolvimento da doença aterosclerótica que tem na sua base fisiopatológica a formação de placas ou ateroma, que são lesões elementares para a doença vascular periférica. ${ }^{31}$

Existem muitos estudos que demonstram a prevalência da hipertensão arterial nos indivíduos com pé diabético, variando entre $14,8 \%$ a $86,3 \% .^{26,32}$ Entretanto, existem poucos artigos com a proposta do atual trabalho de evidenciar, separadamente, a prevalência do pé diabético em grupos de indivíduos diabéticos normotensos e diabéticos hipertensos, fazendo a comparação entre ambos os grupos para compreender se a presença da hipertensão age com fator agravador na prevalência dessa complicação vascular.

\section{CONCLUSÃO}

Nos resultados apresentados pelo presente estudo em relação a distribuição da ocorrência do pé diabético pelo
Brasil, foi observado maior prevalência no estado do Rio Grande do Norte e menor prevalência no estado do Amazonas tanto no grupo de indivíduos diabéticos normotensos, quanto no grupo de indivíduos diabéticos hipertensos. No entanto, há poucos artigos sobre essa temática a nível estadual, o que evidencia a necessidade da realização de mais estudos com o intuito de melhor caracterizar o perfil dessa complicação pelo país.

Através do perfil epidemiológico foi possível constatar que há uma maior prevalência de pé diabético no sexo masculino em indivíduos diabéticos normotensos, enquanto que no grupo de indivíduos diabéticos hipertensos a prevalência foi nos indivíduos do sexo feminino. $E$, nota-se ainda que há aumento da prevalência de ambas comorbidades com o aumento da idade, corroborando com os dados presentes na literatura e nos estudos epidemiológicos existentes. Além disso, o tabagismo e o sobrepeso apresentaram-se como fatores de risco associados, corroborando também com o estabelecido pela literatura.

Por fim, foi constatado que a hipertensão arterial atua como um significante fator agravador para o desenvolvimento do pé diabético. Sendo assim, diante da escassez de estudos que evidenciam tal fato, o presente estudo se mostra relevante, servindo como base tanto na compreensão dos aspectos epidemiológicos acerca da relação entre diabetes e hipertensão, assim como na construção de políticas públicas voltadas para redução da morbimortalidade dessas patologias.

\section{REFERÊNCIAS}

1. International Diabetes Federation. Seventh Edition 2015. International Diabetes Federation. 2015. 144 p.

2. Beagley J, Guariguata L, Weil C, Motala AA. Global estimates of undiagnosed diabetes in adults. Diabetes Res Clin Pract [Internet]. 2014;103(2):150-60. Available from: http://dx.doi.org/10.1016/j.diabres.2013.11.001

3. IBGE. Instituto Brasileiro de Geografia e Estatística. Pesquisa Nacional de Saúde 2013 [Internet]. Instituto Brasileiro de Geografia e Estatística (IBGE). 2014. 181 p. Available from: ftp://ftp.ibge.gov.br/PNS/2013/pns2013.pdf

4. Oliveira JEP de, Júnior RMM, Vencio S. Diretrizes 2017-2018 [Internet]. 2018. 3-383 p. Available from: https://www.diabetes.org.br/profissionais/ images/2017/diretrizes/diretrizes-sbd-2017-2018.pdf

5. Of S, Carediabetes M. American Diabetes Association.Clinical Practice Recommendations.Standars of Medical Care in Diabtes-2017. Diabetes Care. 2017;40:S1. Available from: https://professional.diabetes.org/sites/ professional.diabetes.org/files/media/dc_40_s1_final.pdf

6. Mendonça S de S, Morais J de S, Moura MCGG de. Proposta de um protocolo de avaliação fisioterapêutica para os pés de diabéticos. Fisioter em Mov. 2011;24(2):285-98.

7. Gregg EW, Sattar N, Ali MK. The changing face of diabetes complications. LANCET Diabetes Endocrinol [Internet]. 2016;4(6):537-47. Available from: http://dx.doi.org/10.1016/S2213-8587(16)30010-9

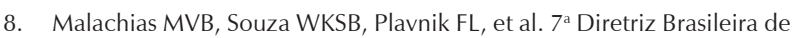
Hipertensão Arterial autores. Arq Bras Cardiol [Internet]. 2016;107:1-103. Available from: http://publicacoes.cardiol.br/2014/diretrizes/2016/05_ HIPERTENSAO_ARTERIAL.pdf

9. Pace AE, Foss MC, Ochoa-Vigo K, Hayashida M. Fatores de risco para complicações em extremidades inferiores de pessoas com diabetes mellitus. Rev Bras Enferm. 2002;55(5):514-21.

10. Moradi Y, Baradaran HR, Djalalinia S, Chinekesh A, Khamseh ME, Dastoorpoor $\mathrm{M}$, et al. Complications of type 2 diabetes in Iranian population: An updated systematic review and meta-analysis. Diabetes Metab Syndr Clin Res Rev [Internet]. 2019;13(3):2300-12. Available from: https://doi. org/10.1016/j.dsx.2019.05.018
11. Vieira-Santos ICR, De Souza WV, De Carvalho EF, De Medeiros MCWC, Nóbrega MGDL, Lima PMS. Prevalência de pé diabético e fatores associados nas unidades de saúde da família da cidade do Recife, Pernambuco, Bra. Cad Saude Publica. 2008;24(12):2861-70.

12. Afroz A, Zhang W, Wei Loh AJ, Jie Lee DX, Billah B. Macro- and microvascular complications and their determinants among people with type 2 diabetes in Bangladesh. Diabetes Metab Syndr Clin Res Rev. 2019;13(5):2939-2946. doi: 10.1016/j.dsx.2019.07.046.

13. Oliveira J de C, Taquary SA dos S, Barbosa A de M, Veronezi RJB. Pé Diabético: Perfil Sociodemográfico e Clínico de Pacientes Hospitalizados. Rev Bras Ciências da Saúde [Internet]. 2018;22(1):15-20. Available from: http://www.periodicos.ufpb.br/index.php/rbcs/article/view/23034/19485

14. Gimenes C, Tinoco TML, Vitta A de, Arca EA, Pessoa-Santos BV, Barrile SR Profile of Hiperdia patients in the municipality of Barra Bonita, Sao Paulo state. Fisioter em Mov. 2016;29(4):731-9.

15. Ochoa-Vigo K, Da Costa Gonçalves Torquato MT, De Souza Silvério IA, De Queiroz FA, De-La-Torre-Ugarte-Guanilo MC, Pace AE. Caracterização de pessoas com diabetes em unidades de atenção primária e secundária em relação a fatores desencadeantes do pé diabético. ACTA Paul Enferm. 2006;19(3):296-303.

16. Brito D, Correia H, Ferreira AV, Jorge $S$, Caniço H. Doença arterial periférica em doentes com diabetes nos cuidados de saúde primários: estudo observacional. Rev Port Clínica Geral. 2017;33(4):290-6.

17. Benjamim G, Pitta B, Castro AA, et al. Perfil dos pacientes portadores de pé diabético atendidos no Hospital Escola José Carneiro e na Unidade de Emergência Armando Lages. J Vasc Bras. 2005;4(1):5-10.

18. Dos Santos Tavares DM, Dias FA, Araújo LR, Pereira GA. Perfil de clientes submetidos a amputações relacionadas ao diabetes mellitus. Rev Bras Enferm. 2009;62(6):825-30.

19. Mané DI, Demba D, Djiby S, et al. Profil clinique et évolutif des lésions de la peau et des parties molles chez les diabétiques en 2017 à la salle de pansement du Centre Marc Sankale de Dakar. Pan Afr Med J. 2019;32:1-11.

20. Sus SIH. Custo de Internações por Pé Diabético no SUS. 2008;523-30. 
21. Antonio M, Nunes P, Resende KF, Castro AA, Benjamin G, Pitta B, et al. Diabeticos. 2006;5(2):123-30.

22. Li X, Xiao T, Wang Y, Gu H, Liu Z, Jiang Y, et al. Incidence, risk factors for amputation among patients with diabetic foot ulcer in a Chinese tertiary hospital. Diabetes Res Clin Pract [Internet]. 2011;93(1):26-30. Available from: http://dx.doi.org/10.1016/j.diabres.2011.03.014

23. Van Houtum WH, Lavery LA. Outcomes associated with diabetes-related amputations in the Netherlands and in the state of California, USA. J Intern Med. 1996;240(4):227-31.

24. Schmidt MI, Hoffmann JF, Diniz MDFS, et al. High prevalence of diabetes and intermediate hyperglycemia - the Brazilian Longitudinal Study of Adult Health (ELSA-Brasil). Diabetol Metab Syndr. 2014;6(1):1-9.

25. Liu M, Zhang W, Yan Z, Yuan X. Smoking increases the risk of diabetic foot amputation: A meta-analysis. Exp Ther Med. 2018;15(2):1680-5.

26. Piccinato $C E$, Cherri J, Moriya T. Hipertensão e doença arterial periférica. Rev Bras Hipertens [Internet]. 2001;8(16):306-15. Available from: http:// departamentos.cardiol.br/dha/revista/8-3/hipertensao.pdf
27. Silva JMTS da, Haddad M do CFL, Rossaneis MA, Vannuchi MTO, Marcon SS. Fatores associados à ulceração nos pés de pessoas com diabetes mellitus residentes em área rural. Rev Gauch Enferm. 2017;38(3):e68767.

28. Goldenberg P, Schenkman S, Franco LJ. Prevalência de diabetes mellitus: diferenças de gênero e igualdade entre os sexos. Rev. bras. epidemiol. [online]. 2003;6(1):18-28.

29. Rossaneis MA, Haddad MCFL, Mathias TAF, Marcon SS. Diferenças entre mulheres e homens diabéticos no autocuidado com os pés e estilo de vida. Rev. Latino-Am. Enfermagem 2016;24:e2761.

30. Simão JB. Um estudo sobre a distribuição das transferências condicionais no setor de saúde no Brasil. Dissertação (Mestrado Profissional em Finanças e Economia) - FGV - Fundação Getúlio Vargas, São Paulo, 2013.

31. Albuquerque MVViana ALDA, Lima LD, Ferreira MP, Fusaro ER, lozzi FL. de et al. Desigualdades regionais na saúde: mudanças observadas no Brasil de 2000 a 2016. Ciênc. saúde coletiva [online]. 2017;22(4):1055-64.

32. Saldiva PHN, Veras M. Gastos públicos com saúde: breve histórico, situação atual e perspectivas futuras. Estudos Avançados. 2018;32(92):47-61. 